\title{
Increasing NER recall with minimal precision loss
}

\author{
Jasper Kuperus \\ Sogeti Nederland B.V. \\ Vianen, The Netherlands \\ Email: mail@jasperkuperus.nl
}

\author{
Cor J. Veenman \\ Netherlands Forensic Institute, Kecida \\ The Hague, The Netherlands \\ Email: c.veenman@nfi.minvenj.nl
}

\author{
Maurice van Keulen \\ University of Twente, EEMCS \\ Enschede, The Netherlands \\ Email: m.vankeulen@utwente.nl
}

\begin{abstract}
Named Entity Recognition (NER) is broadly used as a first step toward the interpretation of text documents. However, for many applications, such as forensic investigation, recall is currently inadequate, leading to loss of potentially important information. Entity class ambiguity cannot be resolved reliably due to the lack of context information or the exploitation thereof. Consequently, entity classification introduces too many errors, leading to severe omissions in answers to forensic queries.

We propose a technique based on multiple candidate labels, effectively postponing decisions for entity classification to query time. Entity resolution exploits user feedback: a user is only asked for feedback on entities relevant to his/her query. Moreover, giving feedback can be stopped anytime when query results are considered good enough. We propose several interaction strategies that obtain increased recall with little loss in precision.
\end{abstract}

\section{INTRODUCTION}

Named Entity Recognition (NER) is a useful technique in many different domains. Although NER has already been researched intensely, it remains a difficult task [1]. One of the main challenges in NER is the ambiguity of the recognized named entities [2]. Examples of ambiguity are whether a numerical value represents a phone number or a social security number (semantic ambiguity), or what the correct boundaries for an entity are (structural ambiguity, e.g., Lake Como or Como). Such ambiguity is often trivial for a human with his cognitive abilities easily interpreting the context [3], but extremely difficult for an automated process.

With the application of forensic investigation in mind, a typical use is network analysis - finding among others relations between people, companies, and addresses, a use well-supported by linking entities occurring in case data. Here, recall is more important than precision: we do not want to lose potentially important information, such as the possibility of a relationship between two persons, which need to be manually confirmed anyway, hence some noise is acceptable.

Most NER solutions identify multiple alternative interpretations of an entity. Machine learning approaches typically assign probabilities to these alternatives, like e.g. Conditional Random Fields (CRFs) [4], [5]. However, most approaches decide on the most probable alternative as the correct classification. For example, a phrase estimated to be either a company or a person name with likelihoods 0.6 and 0.4 respectively does not make company the absolute correct answer, introducing the risk of assigning the wrong type to an entity. These decisions will inevitably result in errors and therefore lower recall.
A method to prevent these extraction errors is to work with multiple candidate classifications, effectively postponing the decision or not making a decision at all. Every identified candidate can be assigned a confidence score that correlates with the probability of this candidate being the correct one [6]. We call Probabilistic Named Entity Recognition (PNER) the approach to keep all possible candidates with their probability.

PNER results in higher recall but also in uncertainty in the data, i.e., lower precision. A method to reduce uncertainty and raise precision is by asking the user to resolve ambiguous situations. We call Targeted Feedback the process of finding the query that results in the highest gain in overall confidence, therefore making user feedback more effective, striving towards a minimal need for user feedback.

Contributions The main contributions of this paper are

- PNER approach enabling postponing and eliminating entity classification decisions, and

- Targeted Feedback strategies for quickly raising data quality with minimal user effort.

$\Rightarrow$ Fewer omissions in answers to (forensic) queries with control over trade-off between user effort and precision.

We first explain our PNER approach in Section II. We then present multiple strategies for Targeted Feedback in Section III that are experimentally compared in Sections IV and V. We end with a discussion of future work and conclusions.

\section{Probabilistic NER}

\section{A. Ambiguity}

There are different kinds of ambiguity that play a role in NER. Van Keulen et al. [3] define three kinds of ambiguity:

- Semantic ambiguity refers to the classification of an entity, does Paris refer to a name or a location?

- Structural ambiguity refers to ambiguity regarding the structure and boundaries of entities, e.g., is the word Lake part of the entity for the location Lake Como?

- Reference ambiguity refers to the question to which real world object an entity refers, e.g., does the location Paris refer to the Paris in France or one of the other 158 Paris instances found in GeoNames [7]?

The problem of reference ambiguity is actively researched, e.g., [8], [9], [10], and our work is complementary to this research. 

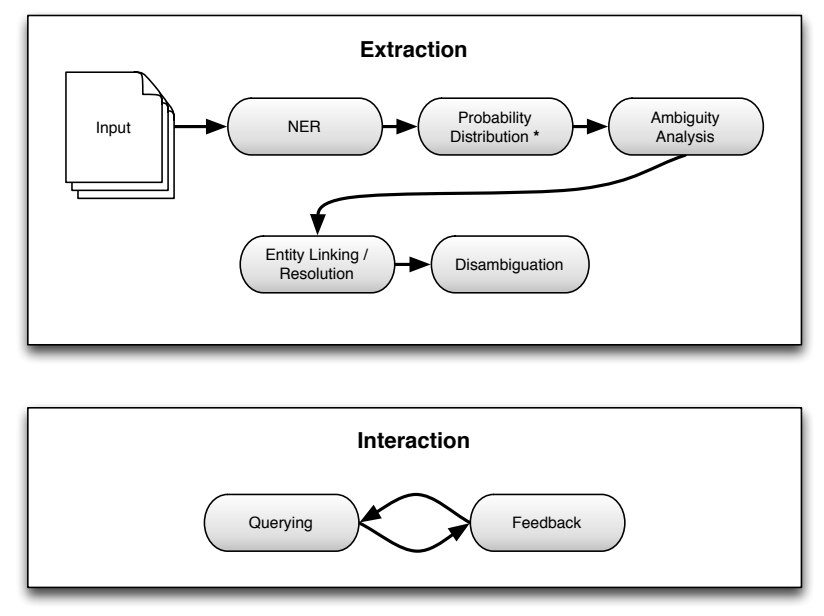

Fig. 1. The "Extraction" and "Interaction" subprocesses of PNER.

\section{B. PNER process}

Since we propose to postpone decisions to query time, we distinguish the subprocesses "Extraction" and "Interaction" (see Figure 1).

1) Extraction subprocess: The extraction subprocess can start in a traditional NER manner. One either adapts an existing NER technique to produce multiple candidates with probabilities or defines a subsequent probability distribution step. The candidate set is first analyzed for structural and semantic ambiguity. Subsequently, the candidates are linked to entries from a knowledge base introducing reference ambiguity. In the remainder of the paper, we focus on semantic and structural ambiguity only. The extraction subprocess ends with a disambiguation step. Although automatic disambiguation can introduce the very errors PNER attempts to avoid, some filtering of obvious cases and redistribution of probabilities given reference knowledge is beneficial.

2) Interaction subprocess: Using probabilistic database technology, the uncertain data produced in extraction can be queried immediately resulting initially in a low quality query result with many alternatives. Targeted Feedback constructs and poses a question to the user whose answer reduces the likelihood or completely eliminates NER candidates, which in turn improve the query result. This process is repeated until the user is satisfied with its quality.

Resolving all ambiguity in a big dataset through user feedback is a labor-intensive task and not the goal of Targeted Feedback. In some situations, resolving ambiguity by $50 \%$ might just be enough for a user to confidently answer his/her question. Also, Targeted Feedback stays within the scope of the query, hence avoiding solving ambiguity concerning entities not relevant to the current information need.

\section{Adapting existing NER techiques for PNER}

As mentioned, it is possible to adapt some existing NER techniques to produce multiple candidates with probabilities.
Although performing hard entity classification, often the absolute decisions are internally based on a probabilistic approach. For example, the Stanford CRF Classifier [11], [12], which we use in our experiments, provides information on the probabilities underlying its classifications.

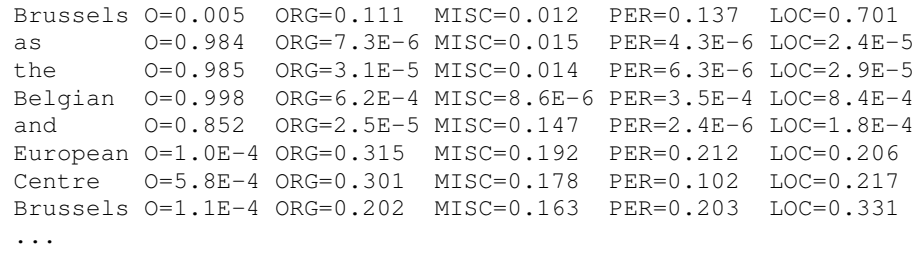

Fig. 2. Stanford CRF Classifier Classification Result Format

As can be seen in Figure 2, it determines per token a probability for each class. These can be directly taken as candidates (semantic ambiguity). A threshold of 0.01 is applied to eliminate marginal candidates. Candidates for structural ambiguity are constructed by detecting subsequent tokens with the same type that combined have a non-marginal probability. The probability of such a multi-token entity can be calculated using Stanford's support for obtaining conditional probabilities and the multiplication axiom [13].

$$
P\left(A_{1} A_{2} \ldots A_{n}\right)=\prod_{i=1}^{n} P\left(A_{i} \mid A_{1} \ldots A_{i-1}\right)
$$

For example, $P($ European Centre=0RG $)=P($ European=0RG $)$. $P($ Centre=0RG | European=0RG $)$.

\section{Probabilistic data model}

The PNER results are label candidates with probabilities. Such results can be readily stored in a probabilistic database. We developed a simple relational model for the experiments in Section IV.

Semantic and structural ambiguity is local, i.e., candidates come in bundles covering a few consecutive words, e.g., the European Centre Brussels we saw earlier. The relational model is based on two tables: Entities and Classifications. The former holds for each entity candidate its id, entity name, boundary (begin and end position), parent boundary (boundary of entity bundle) and structural probability. The latter holds for each entity candidate and each of its possible classes, its id, class, and semantic probability. A candidate for the example bundle is $\langle 42$, European Centre, $(0,15),(0,24), 0.2\rangle$, which could, e.g., have two possible classes: $\langle 42, \mathrm{LOC}, 0.3\rangle$ and $\langle 42, \mathrm{ORG}, 0.5\rangle$.

This relational model cannot express mutual exclusiveness. For example, it assigns non-zero probabilities to impossible worlds such as European $\wedge$ European Centre Brussels. The more complex random variables model solves this problem [14].

\section{TARGETED FEEDBACK}

The extraction subprocess of PNER is fully automatic and, in constrast to regular NER approaches, it retains all possible interpretations. Even though the system may not be able to 


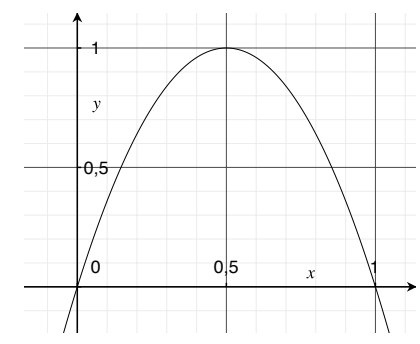

Fig. 3. Graph of MAEF AmbScore function (Equation 2)

provide the correct interpretation with the highest probability, it is careful in not missing the correct interpretation. It will be among all the possibilities, i.e., recall is high. Accordingly, an explosion of possibilities is likely. By employing probabilistic database techniques, this volume of uncertain data can be handled. Clearly, query results will exhibit quite some noise from all these possibilities, i.e., precision is low. If the user is unsatisfied with the precision of the results, he can raise it by giving feedback on ambiguous situations relevant to the query. Feedback is given by answering questions posed by the system that resolve some of the ambiguity. Note that the system does not take automated decisions, the user decides through feedback.

User effort in answering questions is costly and not every feedback raises precision with the same amount. Therefore, user effort can be reduced if the system manages to pose those questions first whose answer would increase precision most. We call this Targeted Feedback. Multiple strategies can be defined for choosing which question to pose next.

Besides these Question Proposal Strategies, we also distinguish Answer Handling Strategies. These vary in what information they derive from the answer, for example, by exploiting opportunities for extrapolation. In the end, Targeted Feedback needs a combination of one of each strategies.

In this paper, we experiment with several baseline and more refined strategies. Ideas for more elaborate and intelligent strategies are discussed in Section VI. The performance of a strategy is defined by both the speed of increase in precision and the moment of the biggest increase.

\section{A. Question Proposal Strategies}

We experiment with the following strategies:

1) Sequential: Baseline strategy that proposes to solve all ambiguous entities in the query answer in the order of their extraction from the original documents.

2) Random: Baseline strategy that randomly picks an ambiguous entity in the query answer.

3) Largest Bundle First (LBF): Structural ambiguity abounds in PNER resulting in many entity bundles. The LBF strategy poses the entity bundle with the most entities to the user first. The intuition is that solving structural and semantic ambiguity of many entities in one question is likely to give a high increase in precision.
4) Most Ambiguous Entity First (MAEF): LBF ignores probabilities. An entity with two candidate classifications $A(0.95)$ and $B(0.05)$ can be considered not very ambiguous. Similarly, a distribution $A(0.9), B(0.07), C(0.03)$ can be considered much less ambiguous than $A(0.44), B(0.32)$, $C(0.24) .{ }^{1}$ The intuition behind MAEF is to pose the entity first that has the highest ambiguity, and if this most ambiguous entity is part of an entity bundle, pose the entire bundle. We measure ambiguity with an ambiguity score function which is a quadratic function with its maximum at 0.5 and zerocrossings at 0 and 1 ( $c l$.prob is the probability of candidate classification $c l$ for entity $e$ ).

$$
\text { AmbScore }(e)=\sum_{c l \in e}-4(\text { cl.prob }-0.5)^{2}+1
$$

5) Most Ambiguous Cluster First (MACF): There are Answer Handling Strategies, such as Statistical (see below), that attempt to extrapolate answers of the user. For example, if the feedback on Amsterdam is very often LOC, then the probability of Amsterdam being LOC in all unresolved entities can be increased. Such an Answer Handling Strategy benefits from being combined with a Question Proposal Strategy that prefers entities containing often occurring entities.

The MACF strategy clusters entities by their name. AmbScore $(c)=\sum_{e \in c}$ AmbScore $(e)$. From the cluster $c$ with the highest score, the entity with the highest score is posed to the user, or the entity bundle if it is part of a bundle.

\section{B. Answer Handling Strategies}

Answer Handling Strategies define what information is derived from the feedback of the user, hence which actions are taken. Possible actions are elimination of candidates or adjusting and redistributing probabilities.

1) BasicDB: This strategy eliminates those entity candidates indicated to be incorrect by the user and assigns a probability of 1.0 to those indicated to be correct. The feedback can also be a new entity or classification, which is inserted into the database with probability 1.0. All other strategies should strictly extend this one, i.e., not refrain from taking these actions.

2) Statistical: This strategy attempts to extrapolate feedback on entities to others with the same name. It keeps track of a counter $c t r_{e, c l}$ per entity-classification combination, for example, (Amsterdam, LOC). Probabilities of all candidates with the same name are redistributed: cl.prob: $=c l . p r o b \cdot\left(c t r_{e, c l}+1\right)$ followed by a normalization of cl.prob for each $e$. For example, a $60 \% / 20 \% / 20 \%$ distribution for Amsterdam being LOC/ORG/MISC is redistributed to $75 \% / 12.5 \% / 12.5 \%$ upon the first feedback that some other Amsterdam is a location.

\section{EXPERIMENTAL SETUP}

\section{A. Data, Queries, and Strategies}

We used the SoNaR [15], [16], [17], [18] corpus, a major reference corpus for contemporary written Dutch (Dec 2011).

\footnotetext{
${ }^{1}$ Note that one of $A, B$, or $C$ can represent the class representing the case 'not an entity at all'.
} 


\begin{tabular}{ll} 
Query & Description \\
\hline A: * & All entities \\
B: *=PER & All persons \\
C: *=EVE & All events \\
D: *=LOC \&2 *Dylan* & $\begin{array}{l}\text { All locations within 2 lines of an entity } \\
\text { containing 'Dylan' }\end{array}$ \\
E: *=PER \&1 USA & All persons within 1 line of 'USA' entities \\
\hline
\end{tabular}

TABLE I

VALIDATION QUERIES

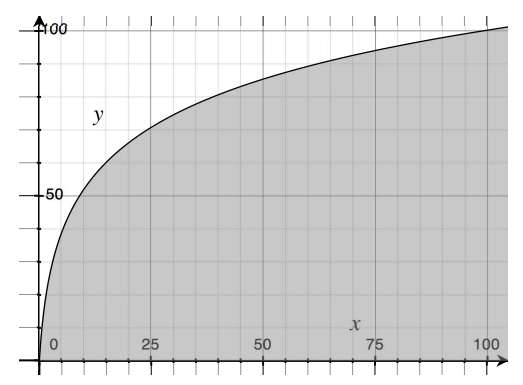

Fig. 4. Strategy Comparison Measure - F-measure Area

worlds. They proved that this is equivalent with replacing 'counting' in the traditional Precision and Recall formulas with a sum of probabilities. Intuitively, it 'counts' an answer by its probability.

We define a third Recall measure, called $E_{100}($ Recall $)$, which does not take into account the probabilities. In this way, it determines the 'coverage', i.e., how many of the correct answers are covered by the candidates in the probabilistic answer, for a large part evaluating a correct setting of a cut-off threshold. Note that although the formula looks the same as for traditional Recall, the numbers of entities here refer to the entire candidate set.

$$
\begin{aligned}
E(\text { Precision }) & =\frac{f(\text { \#correct entities found })}{f(\text { \#total entities found })} \\
E(\text { Recall }) & =\frac{f(\text { \#correct entities found })}{\# \text { total correct } \text { entities }} \\
E_{100}(\text { Recall }) & =\frac{\text { \#correct entities found }}{\text { \#total correct entities }} \\
f(x) & =\sum_{(e, c l) \in x}(\text { e.prob } \cdot \text { cl.prob })
\end{aligned}
$$

Note that for a non-probabilistic result, i.e., without alternatives and a probability of 1 for each result, $E($ Precision $)=$ Precision and $E($ Recall $)=E_{100}($ Recall $)=$ Recall . Furthermore, it is necessary to consider all three measures together as each can be trivially maximized individually judging a different aspect of result quality.

Finally, the performance of a strategy is defined by both the speed of the data quality increase and the moment in time of the biggest increase. No measure yet exists for this purpose, therefore we introduce the $F$-measure Area defined as the area under the $F$-measure graph, as illustrated in Figure 4. We apply this measure to the graph which has the number of questions $n$ on the x-axis and the $F$-measure on the y-axis. The area under the F-measure graph can be calculated using a Riemann sum:

$$
F-\text { measure Area }=\frac{100}{n} \sum_{i=0}^{n} F-\text { measure }_{i}
$$

\section{Results}

\section{A. Initial Query Performance}

Comparing Precision and E(Precision) in Table II shows a higher value for $E($ Precision $)$ for all queries. This tells variants of Precision and Recall for probabilistic data as the expected value of Precision and Recall over all possible 


\begin{tabular}{lccccc} 
Query & Precision & $E($ Precision $)$ & Recall & $E($ Recall $)$ & $E_{100}($ Recall $)$ \\
\hline A & $43.13 \%$ & $61.98 \%$ & $52.17 \%$ & $38.78 \%$ & $87.33 \%$ \\
B & $19.17 \%$ & $28.20 \%$ & $54.80 \%$ & $40.35 \%$ & $95.59 \%$ \\
C & $5.56 \%$ & $14.41 \%$ & $39.76 \%$ & $30.47 \%$ & $65.06 \%$ \\
D & $8.72 \%$ & $15.85 \%$ & $81.82 \%$ & $69.56 \%$ & $95.45 \%$ \\
E & $19.79 \%$ & $21.33 \%$ & $90.48 \%$ & $62.33 \%$ & $95.24 \%$ \\
\hline
\end{tabular}

TABLE II

VALIDATION Queries - Performance

\begin{tabular}{lcc} 
Query & Precision & Recall \\
\hline A & $61.61 \%$ & $57.60 \%$ \\
B & $64.72 \%$ & $66.51 \%$ \\
C & $88.89 \%$ & $38.55 \%$ \\
D & $31.36 \%$ & $84.09 \%$ \\
E & $3.19 \%$ & $95.24 \%$ \\
\hline
\end{tabular}

TABLE III

VALIDATION QUERIES - STANFORD NER

us that a considerable amount of correct answers in the dataset are not assigned the highest probability among their alternatives and are therefore not extracted with traditional NER. Comparing Recall with $E_{100}$ (Recall) shows that PNER manages to extract much more of the correct answers among its alternatives, correct answers that would be missed with the absolute decision making of traditional NER.

The initial Precision and E(Precision) values seem quite low. However, Kuperus [14] provides an extensive explanation on how these values relate to the different kinds of ambiguity and how this results in the shown Precision values.

\section{B. Traditional NER}

Our PNER approach introduces an additional dimension to the probabilistic results of Stanford NER [11], structural ambiguity. Therefore, Precision and Recall in Table II do not reflect traditional NER. Table III shows the performance of Stanford as-is. Comparing the Recall of Stanford with $E_{100}($ Recall) shows that PNER has a significantly better coverage of the correct answers than traditional NER, adding up to a difference over $\mathbf{2 9 \%}$ for queries $\mathbf{A}$ and $\mathbf{B}$.

\section{Strategy Performance}

We have observed the general trend that for almost all combinations of strategies and queries both Precision and Recall approach $100 \%$ (not shown). This is due to the high initial $E_{100}($ Recall $)$ and the fact that a user can introduce new entities and classifications.

Table IV presents a performance comparison for all strategy combinations. The baseline strategy Sequential is outperformed in almost all cases. The other baseline strategy Random proved harder to beat.

Although the MACF strategy is introduced mainly as enhancement of MAEF and meant to be combined with Statistical, it is in most cases outperformed by both LBF and MAEF. The reason for this can be found in the fact that the query

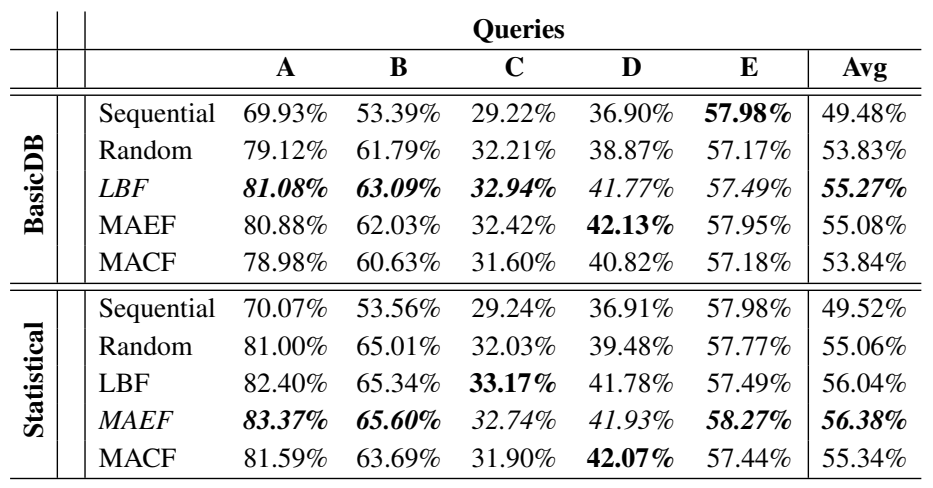

TABLE IV

Strategy Result MAtriX - F-MeAsure AREA

results are highly heterogeneous, which Kuperus explains in depth in [14].

Figure 5 compares Answer Handling Strategies BasicDB and Statistical on a heterogeneous as well as a more homogeneous query (return all occurrences of "Sri Lanka"). The benefit of Statistical over BasicDB is more significant in the latter case. It can be concluded that in heterogeneous situations clustering and extrapolating probabilities merely on the name of an entity is less effective.

\section{DISCUSSION}

In this paper, we presented and experiment with only the top of the iceberg of possible strategies for Targeted Feedback. A detailed list of proposals for more advanced strategies can be found in [14]. We like to mention three:

- Context analysis The presented strategies merely look at entities, their name, and their possible classifications. Analyzing the context, e.g. POS tags of the surrounding text, may be a useful technique in Answer Handler Strategies, extrapolating the given answer not only to entities having similar name, but also similar context.

- Similarity The Statistical strategy redistributes probabilities of entities having similar name. Similarity can be established on many other criteria. By defining a similarity function based on other features, other patterns of extrapolation can be achieved, both in Answer Handling as well as Question Proposal.

- Extrapolation scope The Statistical strategy redistributes probabilities of entities throughout the whole dataset. It is likely that it is not justified to extrapolate answers to such a wide scope. Limiting the scope of extrapolation may boost performance.

\section{CONCLUSIONS}

Comparing Recall for regular NER to the $E_{100}($ Recall) of PNER, it can be concluded that PNER shows a significantly bigger coverage of the correct answer, adding up to a difference over 29\% for the whole dataset. Comparing regular NER Precision and E(Precision) of PNER for the whole dataset, it shows that regular NER does not weigh up to weighing 


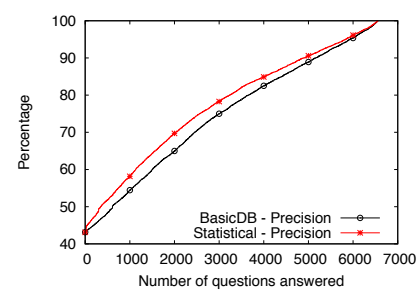

(a)

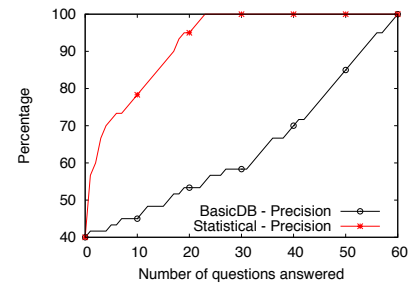

(e)

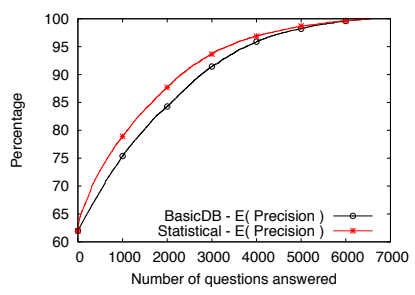

(b)

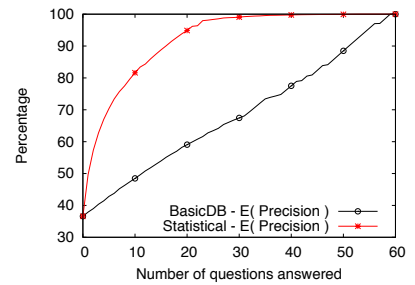

(f)

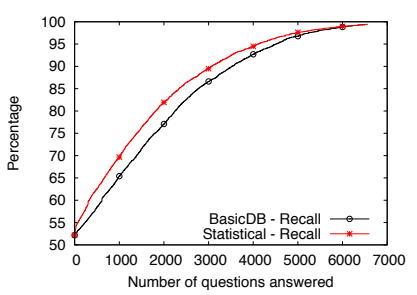

(c)

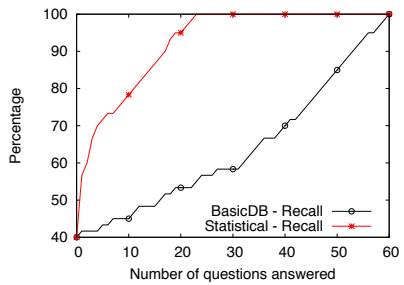

(g)

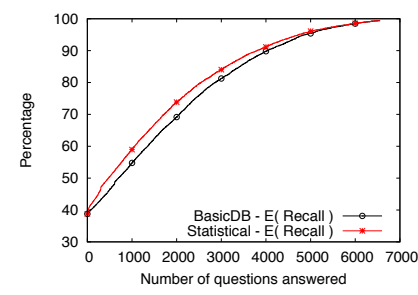

(d)

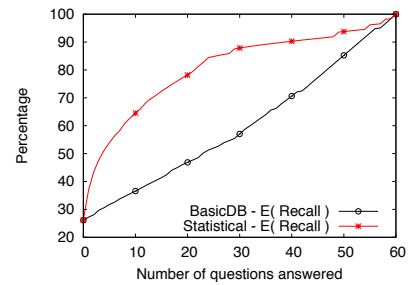

(h)

Fig. 5. BasicDB vs. Statistical using MACF; (a)-(d) Query A; (e)-(h) Query "Sri Lanka"

each correct answer by its probability. In other words, a considerable amount of entities would be lost when classified based on the highest entity class probability.

Targeted Feedback aims on solving the introduced ambiguity by posing the user targeted questions and attempting to learn from the answers provided by the user. When deploying Targeted Feedback, eventually both Precision and Recall approach $100 \%$, proving convergence of the method. However, the time and effort of the user are costly. If the user has to inspect each reported entity before the desired data quality is reached, the user might rather choose to perform NER manually.

The implemented Targeted Feedback strategies do not show spectacular performance gain. Whether PNER is more useful than regular NER depends on the application. Clearly, there is a significant amount of ambiguity in the results. Further study is needed to find out whether the required user interaction is feasible in real forensic queries. Also secondary analysis, such as entity based document linking, must be tested for its robustness against noise.

Finally, it can be concluded that PNER in combination with Targeted Feedback shows real potential compared to regular NER. The initial PNER results cover significantly more correct answers, which would be discarded during regular NER, and using Targeted Feedback, the introduced ambiguity can be resolved and data quality in terms of both Recall and Precision eventually approach $100 \%$.

\section{REFERENCES}

[1] D. F. Bon, "Domain specific named entity recognition for forensic intelligence," 2011, University of Amsterdam.

[2] N. Wacholder, Y. Ravin, and M. Choi, "Disambiguation of Proper Names in Text," in Proc. of the 5th Conf. on Applied Natural Language Processing, 1997.

[3] M. van Keulen and M. B. Habib, "Named Entity Extraction and Disambiguation: The Reinforcement Effect," in Proc. of the 5th Int'l Workshop on Management of Uncertain Data, MUD 2011, 2011.

[4] R. Klinger and K. Tomanek, Classical Probabilistic Models and Conditional Random Fields, ser. Algorithm Engineering Report. Dortmund University of Technology, 2007, no. TR07-2-013, ISSN 1864-4503.
[5] H. W. Wallach, "Conditional Random Fields: An Introduction," Rapport technique MS-CIS-04-21, University of Pennsylvania, vol. 50, 2004.

[6] R. Gupta and S. Sarawagi, "Creating probabilistic databases from information extraction models," in Proc. of the VLDB Endowment, 2006.

[7] GeoNames, "GeoNames," Available at: http://www.geonames.org/, Last visited Nov. 2011.

[8] J. Hoffart, M. Yosef, I. Bordino, H. Fürstenau, M. Pinkal, M. Spaniol, B. Taneva, S. Thater, and G. Weikum, "Robust Disambiguation of Named Entities in Text," in Proc. of EMNLP, 2011.

[9] Max Planck Institute for Informatics, "AIDA Web Interface," Available at: https://d5gate.ag5.mpi-sb.mpg.de/webaida/, Last visited Nov. 2011.

[10] M. A. Yosef, J. Hoffart, I. Bordino, M. Spaniol, and G. Weikum, "AIDA: An Online Tool for Accurate Disambiguation of Named Entities in Text and Tables," in Proc. of the VLDB Endowment, 2011.

[11] Stanford University: Natural Language Processing, "Stanford Named Entity Recognizer," Available at: http://nlp.stanford.edu/software/CRFNER.shtml, Last visited Mar. 2012.

[12] J. Finkel, T. Grenager, and C. Manning, "Incorporating Non-local Information into Information Extraction Systems by Gibbs Sampling," in Proc. of the 43rd Annual Meeting on Association for Computational Linguistics, 2005.

[13] J. Kalbfleisch, Probability and Statistical Inference, Volume 1: Probability. Springer, 1985.

[14] J. Kuperus, "Catching criminals by chance: a probabilistic approach to named entity recognition using targeted feedback," Master's thesis, University of Twente, 2012, http://eprints.eemcs.utwente.n1/21948.

[15] M. Reynaert, N. Oostdijk, O. De Clercq, H. Heuvel, and F. Jong, "Balancing SoNaR: IPR versus processing issues in a 500-millionword written Dutch reference corpus," in Proc. of the 7th Conf. on International Language Resources and Evaluation (LREC), 2010.

[16] Nederlandse Taalunie, "STEVIN: Spraak- en Taaltechnologische Essentiële Voorzieningen in het Nederlands," Available at: http://taalunieversum.org/taal/technologie/stevin/, Last visited Oct. 2011.

[17] Centre for Language and Speech Technology, "SoNaR: STEVIN Nederlandstalig Referentiecorpus," Available at: http://lands.let.ru.nl/projects/SoNaR/, Last visited Oct. 2011.

[18] I. Schuurman, V. Hoste, and P. Monachesi, "Interacting Semantic Layers of Annotation in SoNaR, a Reference Corpus of Contemporary Written Dutch," in Proc. of the 7th Int'l Conf. on Linguistic Resources and Evaluation (LREC), 2010.

[19] D. Jurafsky and J. H. Martin, Speech And Language Processing. Pearson Education, 2009.

[20] R. Baeza-Yates, B. Ribeiro-Neto et al., Modern Information Retrieval. ACM press New York, 1999.

[21] M. van Keulen and A. de Keijzer, "Qualitative Effects of Knowledge Rules and User Feedback in Probabilistic Data Integration," The VLDB Journal, 2009. 